\title{
Prescription medication changes following direct-to-consumer personal genomic testing: findings from the Impact of Personal Genomics (PGen) Study
}

\author{
Deanna Alexis Carere, ScD, CGC1, Tyler J. VanderWeele, PhD², Jason L. Vassy, MD, MPH, SM ${ }^{3,4,5}$, \\ Cathelijne H. van der Wouden, PharmD ${ }^{6}$, J. Scott Roberts, $\mathrm{PhD}^{7}$, Peter Kraft, $\mathrm{PhD}^{2}$ and \\ Robert C. Green, MD, MPH ${ }^{5,8,9}$; for the PGen Study Group
}

Purpose: To measure the frequency of prescription medication changes following direct-to-consumer personal genomic testing (DTC-PGT) and their association with the pharmacogenomic results received.

Methods: New DTC-PGT customers were enrolled in 2012 and completed surveys prior to the return of results and 6 months after results; DTC-PGT results were linked to survey data. "Atypical response" pharmacogenomic results were defined as those indicating an increase or decrease in risk of an adverse drug event or likelihood of therapeutic benefit. At follow-up, participants reported prescription medication changes and health-care provider consultation.

Results: Follow-up data were available from 961 participants, of whom $54(5.6 \%)$ reported changing a medication they were taking or starting a new medication due to their DTC-PGT results. Of these,
45 (83.3\%) reported consulting with a health-care provider regarding the change. Pharmacogenomic results were available for 961 participants, of which 875 (91.2\%) received one or more atypical response results. For each such result received, the odds of reporting a prescription medication change increased 1.57 times ( $95 \%$ confidence interval $=1.17,2.11$ ).

Conclusion: Receipt of pharmacogenomic results indicating an atypical drug response is common with DTC-PGT and is associated with prescription medication changes; however, fewer than $1 \%$ of consumers report unsupervised changes at 6 months after testing.

Genet Med advance online publication 22 September 2016

Key Words: direct-to-consumer genetic testing; pharmacogenomics; pharmacogenetics; personal genomic testing; prescription medications

\section{INTRODUCTION}

Direct-to-consumer (DTC) personal genomic testing (PGT), whereby individuals purchase specific genetic analyses directly from private companies, has the potential to prompt inappropriate use of health-care services. ${ }^{1}$ In studies, however, this concern has not been borne out. ${ }^{2,3}$ One likely reason is that most health services are subject to gate-keeping, ${ }^{4}$ and consumers can rarely access care without clinician involvement.

An exception to this de facto regulation mechanism lies in DTC pharmacogenomic testing. When the US Food and Drug Administration (FDA) sent a warning letter to $23 \mathrm{andMe}$, Inc. (23andMe), in November 2013, the agency speculated that the DTC-PGT company's customers might use their results to "self-manage their treatments through dose changes or even [abandonment of] therapies" ${ }^{5}$ without contacting a physician or pharmacist. With the exception of a single cross-sectional survey that reported posttest medication changes in $<5 \%$ of
DTC-PGT consumers, ${ }^{6}$ no empirical evidence exists to evaluate the validity of the FDA's concerns; moreover, no study has evaluated the relationship between consumers' actual pharmacogenomic test results and post-PGT prescription medication changes.

We present data from the Impact of Personal Genomics (PGen) Study, ${ }^{7}$ a longitudinal study of DTC-PGT customers of 23andMe and Pathway Genomics Corp. (Pathway) surveyed prior to and 6 months following the return of results. Our analytic goals were threefold: (i) to describe the frequency and types of pharmacogenomic results received by DTC-PGT consumers; (ii) to describe the frequency and types of postPGT prescription medication changes reported by DTC-PGT consumers; and (iii) to test the hypothesis that receipt of pharmacogenomic results predicting an atypical drug response is associated with post-PGT prescription medication changes. In light of a previously documented association between receipt

\footnotetext{
The last two authors contributed equally to this work.

See acknowledgements for list of nonauthor members of the PGen Study Group.

${ }^{1}$ Department of Pathology and Molecular Medicine, McMaster University, Hamilton, Ontario, Canada; ${ }^{2}$ Department of Epidemiology, Harvard School of Public Health, Boston, Massachusetts, USA; ${ }^{3}$ Division of General Medicine and Primary Care, Department of Medicine, Brigham and Women's Hospital, Boston, Massachusetts, USA; ${ }^{4}$ VA Boston Healthcare System, Boston, Massachusetts, USA; ${ }^{5}$ Harvard Medical School, Boston, Massachusetts, USA; ${ }^{6}$ Department of Clinical Pharmacy and Toxicology, Leiden University Medical Center, Leiden, The Netherlands; ${ }^{7}$ Department of Health Behavior and Health Education, University of Michigan School of Public Health, Ann Arbor, Michigan, USA; ${ }^{8}$ Division of Genetics, Department of Medicine, Brigham and Women's Hospital, Boston, Massachusetts, USA; ${ }^{9}$ Broad Institute, Cambridge, Massachusetts, USA.

Correspondence: Robert C. Green (rcgreen@genetics.med.harvard.edu)
} 
of DTC pharmacogenomic information and post-PGT health services usage, ${ }^{8}$ we also used secondary analyses to evaluate the association between receipt of atypical drug response results and three measures of health services usage.

\section{MATERIALS AND METHODS}

\section{The PGen Study}

The PGen Study was initiated in 2011 by academic researchers at Harvard Medical School/Brigham and Women's Hospital (Boston, MA) and the University of Michigan School of Public Health (Ann Arbor, MI) and by industry scientists at 23andMe 9 (Mountain View, CA) and Pathway ${ }^{10}$ (San Diego, CA). The study was approved by the Partners Human Research Committee and the University of Michigan School of Public Health Institutional Review Board.

New customers were recruited between March and July 2012. E-mails with information about the study and an invitation to participate were sent by $23 \mathrm{andMe}$ to a consecutive series of 3,900 customers who ordered the company's service during this time period and who had previously submitted general consent to be contacted about research opportunities; of these, 1,249 (32.0\%) provided online consent to the PGen Study. Separately, Pathway placed a banner advertisement for their services and the PGen Study on their webpage and sent e-mails to approximately 30,000 members of PatientsLikeMe, a health-based social networking site. ${ }^{11}$ In these communications, Pathway's PGT service was offered at a subsidized price of $\$ 25$. After placing an order for PGT through one of these channels, customers were directed to a webpage inviting them to participate in the PGen Study. A total of 589 Pathway customers provided online consent.

Of the 1,838 individuals who consented, 1,648 (23andMe $=1,085$; Pathway $=563)$ completed a baseline survey prior to receiving their PGT results. Eligibility criteria for follow-up requiring receipt and access of health-related results within the study period were met by 1,464 participants $(23 \mathrm{andMe}=947$; Pathway $=517)$. Follow-up surveys were administered 2 weeks after results $\left(n=1,046\right.$; response rate $\left.{ }^{12}=71.4 \%\right)$ and 6 months after results $(1,042 ; 71.1 \%)$. Results were returned to customers by the companies and then transferred to academic researchers and linked to survey data. Complete details of the collaborative arrangement with the companies, participant recruitment, inclusion/exclusion criteria, and data collection (including a nonresponse bias analysis and full versions of the surveys) are published elsewhere.,13 Throughout the PGen Study, each company was represented by one research scientist; that scientist was invited to comment on papers in progress. A presubmission manuscript was sent to each representative for review and comment, but final discretion was allocated to the writing group, particularly the first author (D.A.C.) and the PGen Study's co-principal investigators (J.S.R., R.C.G.).

\section{Survey instruments}

At baseline, we measured age, race/ethnicity, ${ }^{14}$ gender, income, education, self-reported health, ${ }^{15}$ health insurance status, current prescription medication use (yes/no in seven categories), and interest in obtaining pharmacogenomic information (three ordinal categories). Among participants who reported undergoing a physical examination in the past 2 years, we measured the number of self-reported health-care visits during the past year (shown to correlate strongly with medical recordsbased measures of health-care visits ${ }^{16}$ ).

At 6-month follow-up, participants reported changes to their prescription medications "as a result of seeing [their company] results" (yes/no in five categories), whether they had consulted a health-care provider before making the change, and what had prompted them to make the change(s) (free-text response). "Health-care provider" was not further defined; therefore, reported consultations could capture interactions with nonphysicians, alternative medicine practitioners, or other nonprescribing professionals (e.g., nurses). Participants were also asked: "Do you think you will use your [company] results to guide your future use of medication?" (yes/no/do not know).

Finally, participants reported three post-PGT outcomes previously shown by Bloss et al. ${ }^{8}$ to be associated with receipt of DTC pharmacogenomic information: sharing of PGT results with a health-care provider (yes/no); follow-up tests, medical examinations, or procedures ordered on the basis of their PGT results (yes/no); and number of health-care visits since PGT (6- month interval). The first of these outcomes was measured with a survey item that asked participants with whom they had shared their results (nine available responses, including three health-care provider categories: "Primary care provider," "Genetics specialist (e.g., genetic counselor, clinical geneticist)," and "Other medical professional"). If participants selected "Other medical professional," they were asked to identify the type of medical professional from a list. "Primary Care Provider" was not further defined in the survey and therefore was open to participant interpretation (i.e., may have included nonphysician medical practitioners and alternative medicine providers). The second of these outcomes was measured with a single survey item that read: "As a result of seeing your genetic information from [company], have you had any tests, medical exams, or procedures?" The inclusive wording of this item was designed to capture services received in response to any PGT result (e.g., disease risk estimates, genetic carrier status) and not only pharmacogenomic information. Note that Bloss et al. evaluated the impact of receiving any DTC pharmacogenomic information (without consideration of the content of that information) on health services usage within a sample of consumers who were randomized to either receive or not receive pharmacogenomic results. Here, we instead evaluated the impact of receiving atypical pharmacogenomic results within a sample of consumers who all received pharmacogenomic reports.

\section{PGT results}

Participants received pharmacogenomic information within their comprehensive PGT reports. 23andMe customers received up to eight pharmacogenomic results, presented as a relative risk for each adverse outcome (or relative benefit for treatment 
efficacy traits) compared with someone in the general population of the same ethnicity. Pathway customers received up to nine pharmacogenomic results, presented as either "normal," "beneficial effect," or "adverse effect."

\section{Statistical analyses}

Data were obtained from PGen Study participants who submitted both baseline and 6-month surveys and who had complete data for age, gender, race/ethnicity, education, self-reported health, interest in pharmacogenomic PGT results, baseline prescription medication use, and changes to prescription medications after PGT. Analyses were restricted to participants whose pharmacogenomic results were available to researchers. Pharmacogenomic results were classified as either atypical response (increased relative risk of an adverse drug event; increased or decreased likelihood of therapeutic benefit) or typical response (average risk of an adverse event; typical therapeutic response). We summarized baseline participant characteristics, frequency of atypical response results, and post-PGT prescription medication changes, using descriptive statistics.

We performed logistic regression of "reporting any change to a prescription medication" (and in separate models, each type of change) on the number of atypical response pharmacogenomic results received. We used similar models to test the association of atypical response results with (i) sharing PGT results with a health-care provider and (ii) undergoing additional tests, examinations, or procedures post-PGT. Finally, we performed linear regression of "change in number of health-care visits from prePGT to post-PGT" on number of atypical response pharmacogenomic results received. The number of visits in the past year reported at baseline was divided by two for this analysis.

Analyses were conducted using SAS 9.3 (SAS Institute, Cary, NC), and models were fitted using PROC GLM (linear regression) and PROC LOGISTIC (logistic regression). Models of post-PGT outcomes were adjusted for age, gender, race/ethnicity, education, health insurance status, PGT company, selfreported health, and baseline interest in pharmacogenomic results. The model for change in mean health-care visits was additionally adjusted for number of health-care visits reported at baseline (divided by two). Because household income is associated with both health services utilization ${ }^{17}$ and prescription medication adherence, ${ }^{18}$ we evaluated the impact of adjusting for income for participants for whom these data were available. Statistical significance was set at $P<0.05$.

\section{Qualitative analyses}

Free-text responses to the question about medication changes were reviewed by DAC for two themes: use of pharmacogenomic results to guide medication changes and use of other PGT results to guide medication changes.

\section{RESULTS}

\section{Participants and pharmacogenomic results}

Thirty-nine participants were excluded due to missing data on self-reported health $(n=2)$ or post-PGT prescription medication changes $(n=37)$. An additional 42 participants (all from 23 andMe) were excluded because their pharmacogenomic results were not available to researchers. These participants had one or more genetic relatives with a linked 23andMe account (i.e., relatives who had ordered 23andMe testing prior to or following the PGen Study and indicated a familial relationship during registration). Given the possibility of revealing personal information about individuals who did not consent to the PGen Study, the genetic results of participants with linked relatives were withheld by 23 andMe. Baseline sociodemographic characteristics of the final sample $(N=961)$ are shown in Table 1. Of these 961 participants, $876(91.2 \%)$ received at least one result indicating atypical drug response (mean $=1.81 \pm 1.04$, range $=0-5)($ Table 2$)$.

\section{Post-PGT prescription medication changes}

Fifty-four participants (5.6\% of 961) reported changing a prescription medication they were already using or starting a new medication by 6-month follow-up (Table 3). Of these, $46(85.2 \%)$ reported consulting a health-care provider before doing so. Among those who reported baseline use of a prescription medication within the measured categories $(n=537), 39$ (7.3\%) reported a change to a prescription medication they were already using and 34 of them $(87.2 \%)$ reported consulting a health-care provider.

We observed a significant association between atypical response pharmacogenomic results and reported prescription medication changes (Table 4): for each atypical response result received, participants had 1.57 times greater odds $(95 \% \mathrm{CI}=$ 1.17-2.11) of reporting a change to a prescription medication within 6 months of undergoing PGT. This relationship held for all specific types of medication changes with the exception of increasing the dose of a medication (Table 5). Because of the small number of events per change category and concerns about model instability, we adjusted only for variables that were significantly associated with changing a prescription medication in the main model and with PGT company.

Looking ahead, 166 participants (17.3\% of 961) did not think they would use their results to guide their future use of medication, whereas 471 (49.0\%) thought they would and 324 (33.7\%) did not know. Within these three groups (no/yes/not sure), the frequency of having received at least one atypical drug response result was $85.5,94.5$, and $89.2 \%$, respectively, whereas the frequency of having already reported making a change to a prescription medication at 6-month follow-up was $0.6,10.8$, and $0.6 \%$, respectively.

\section{Post-PGT health services usage}

Forty-nine participants did not report whether or with whom they had discussed their results. Of the remainder, 336 (36.8\% of 912) reported sharing with a health-care provider, including $260(28.5 \%)$ with a primary care provider, 31 (3.4\%) with a genetics specialist, and $157(17.2 \%)$ with a different medical professional. Other medical professionals with whom participants reported sharing their results included a physician assistant, a 
Table 1 Baseline characteristics of PGen Study participants $(N=961)$

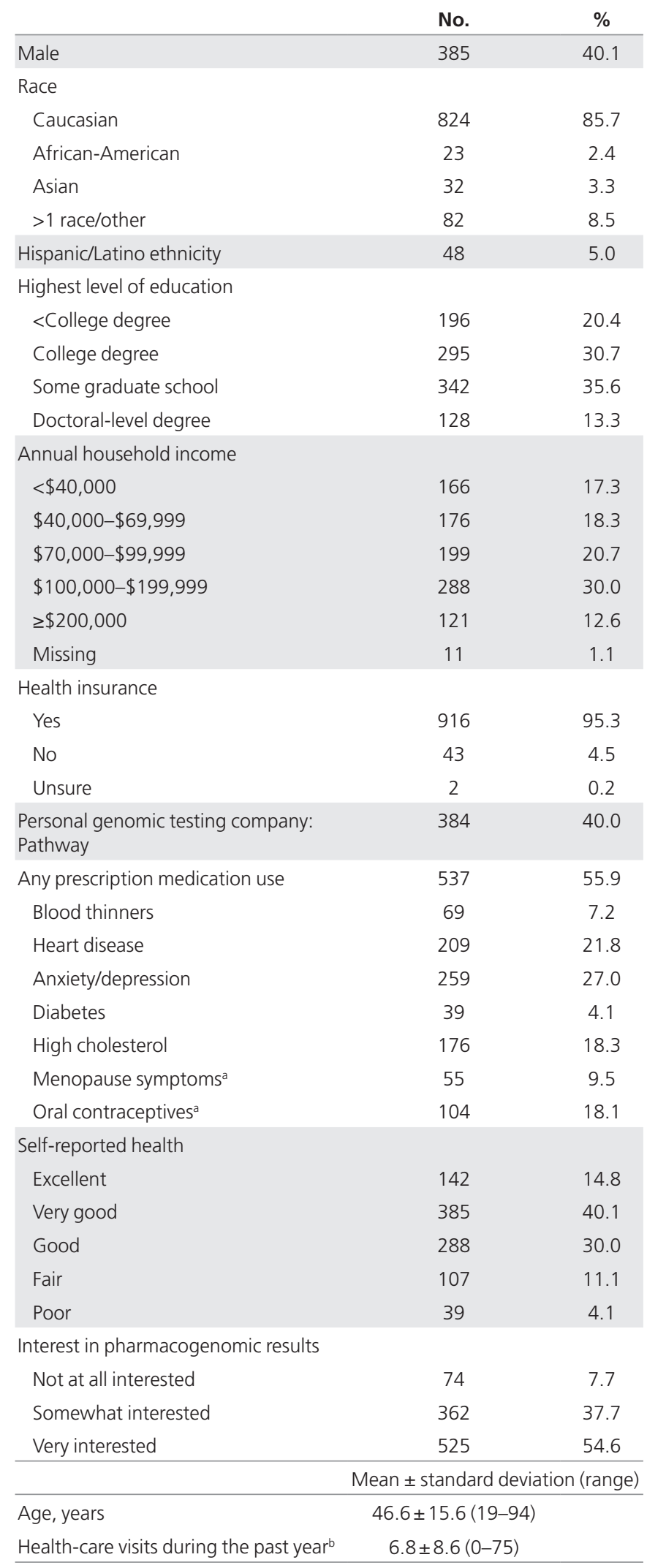

aEvaluated among women only $(n=576)$. ${ }^{b} n=752$; includes only participants who reported having a physical examination within the past 2 years. nurse, or a medical assistant $(n=32)$; an obstetrician/gynecologist (17); an oncologist (10); a surgeon (10); an anesthesiologist (4); a pediatrician/child's physician (4); a nutritionist (3); a reproductive endocrinologist (2); and other specialist (115, encompassing a variety of free-form responses).

Follow-up tests, examinations, and procedures based on PGT results were reported by 105 (10.9\% of 961) participants. Among the 752 participants for whom data were available at both time points, the median number of healthcare visits during the 6 months both prior to and following PGT was 2.0 (Wilcoxon signed rank test: S-statistic $=567$; $P=0.90$ ). There was no significant association between the number of positive pharmacogenomic results received and any of these post-PGT outcomes after multivariable adjustment (Table 4).

\section{Qualitative data and case studies}

Thirty-eight of the participants who reported changing a prescription medication provided free-text responses regarding their motivations (Supplementary Table S1 online). Of these, 15 made reference to a pharmacogenomic result motivating their medication change. For example, a 59-year-old at increased risk for methotrexate toxicity reported:

Doctor had planned on putting me on methotrexate for inflammation at my next office visit, but once he [saw] the genome study he decided to put me on a different drug instead, which has worked well without the side effects.

Four responses attributed the change to some other personal genomic testing result. For example, a 46-year-old whose PGT results indicated an elevated risk for coronary heart disease reported:

The heart disease possibility [led] to the comprehensive blood panel test, which showed I'm off the charts when it comes to cholesterol. This resulted in prescriptions for Crestor [rosuvastatin] and Niaspan [niacin].

Twenty responses did not reference a specific PGT result. As further examples of the types of medication changes reported, two cases are presented:

Case 1: Ms. $\mathrm{X}$ is a Caucasian woman in her 50 s who reported baseline use of medication for heart disease, anxiety/depression, diabetes, high cholesterol, and menopause symptoms. She also reported a family history of heart disease and high cholesterol. Ms. X received two atypical pharmacogenomic results: an increased likelihood of statin therapeutic benefit and an increased risk of statin-induced myopathy. She explains: "Since I am sensitive to statins but have high cholesterol/triglycerides, I decided to cut my TriCor [fenofibrate] dosage to every other day." She did not consult with her physician before making this change. 


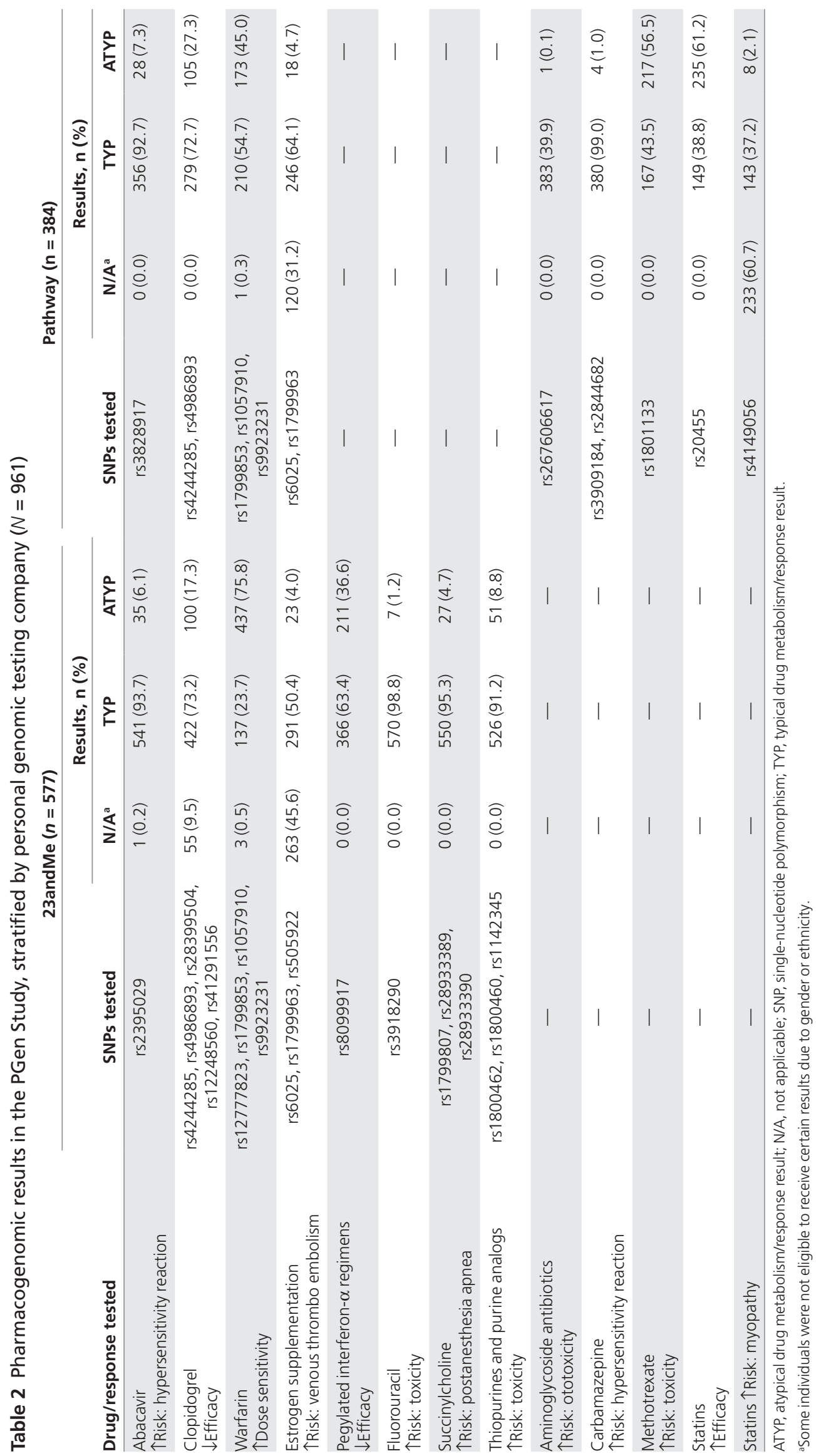


Table 3 Reported changes to prescription medications 6 months after personal genomic testing stratified by health-care provider consultation status $(N=961)$

\begin{tabular}{|c|c|c|c|c|c|c|}
\hline & \multicolumn{2}{|c|}{$\begin{array}{l}\text { Consulted } \\
\text { HCP }\end{array}$} & \multicolumn{2}{|c|}{$\begin{array}{c}\text { Did not } \\
\text { consult HCP }\end{array}$} & \multicolumn{2}{|c|}{ Total } \\
\hline & $n$ & $\%$ & $n$ & $\%$ & $N$ & $\%$ \\
\hline $\begin{array}{l}\text { Any reported change to a } \\
\text { prescription medication }\end{array}$ & 45 & 4.7 & $9^{a}$ & 0.9 & 54 & 5.6 \\
\hline $\begin{array}{l}\text { Started taking a new } \\
\text { medication }\end{array}$ & 13 & 1.4 & 0 & 0.0 & 13 & 1.4 \\
\hline $\begin{array}{l}\text { Stopped taking a current } \\
\text { medication }\end{array}$ & 23 & 2.4 & 5 & 0.5 & 28 & 2.9 \\
\hline $\begin{array}{l}\text { Increased the dose of a } \\
\text { current medication }\end{array}$ & 5 & 0.5 & 0 & 0.0 & 5 & 0.5 \\
\hline $\begin{array}{l}\text { Decreased the dose of a } \\
\text { current medication }\end{array}$ & 11 & 1.1 & 2 & 0.2 & 13 & 1.4 \\
\hline $\begin{array}{l}\text { Switched from one } \\
\text { medication to another }\end{array}$ & 14 & 1.5 & 1 & 0.1 & 15 & 1.6 \\
\hline
\end{tabular}

$\mathrm{HCP}$, health-care provider

ane participant who reported making a change to a prescription medication without consulting a HCP did not indicate that any of these specific changes were made.

Table 4 Regression of post-PGT health-care outcomes on number of atypical response pharmacogenomics results received

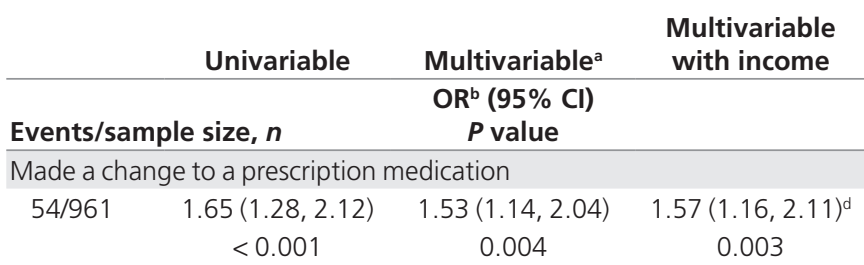

Shared personal genomic testing results with a health-care provider

$336 / 912 \quad 1.24(1.09,1.41) \quad 1.12(0.97,1.30) \quad 1.14(0.99,1.32)^{\mathrm{e}}$
0.001
0.11
0.07

Had tests, examinations, or procedures as a result of genomic information

$105 / 961 \quad 1.14(0.94,1.39) \quad 1.08(0.88,1.33) \quad 1.07(0.87,1.33)^{d}$

\begin{tabular}{|c|c|c|c|}
\hline & 0.17 & 0.47 & 0.51 \\
\hline Sample size & & $\beta^{\mathrm{b}}(95 \% \mathrm{Cl}) P$ value & \\
\hline \multicolumn{4}{|c|}{ Change in mean number of health-care visits per yearc } \\
\hline 752 & $0.05(-0.29,0.40)$ & $0.03(-0.32,0.38)$ & $0.07(-0.29,0.42)^{f}$ \\
\hline & 0.76 & 0.88 & 0.71 \\
\hline
\end{tabular}

$\mathrm{Cl}$, confidence interval; $\mathrm{OR}$, odds ratio; $\mathrm{PGT}$, personal genomic testing.

aMultivariable models adjusted for baseline age, gender, race, ethnicity, education, health insurance status, self-reported health, interest in pharmacogenomic information, and PGT company. ${ }^{\mathrm{b} O d d s}$ ratio/mean change per positive pharmacogenomic test result received. 'Additionally adjusted for frequency of health-care visits at baseline. ${ }^{d} n=950$ due to missing data on income for 11 participants. ${ }^{e} n=902$ due to missing data on income for 10 participants. ${ }^{n} n=744$ due to missing data on income for 8 participants.

Case 2. Ms. Y is a Caucasian woman in her 30 s who reported baseline use of oral contraceptives. Ms. Y received three atypical pharmacogenomic results: increased metabolism of warfarin; reduced efficacy of treatment for Hepatitis C; and increased risk of venous thromboembolism with estrogen supplementation. She explains that she "stopped taking birth control for a trial period and [is] now starting back on a lower dose due to increased risk for venous thromboembolism." She reports consulting with her physician regarding this change.

\section{DISCUSSION}

Among DTC-PGT customers enrolled in the PGen Study, $91.2 \%$ received at least one pharmacogenomic result indicating atypical drug metabolism, a proportion consistent with prior estimates. For example, a 2014 study $^{19}$ found that among 9,589 hospital patients offered preemptive genotyping for 5 druggene interactions, 8,760 (91.4\%) received at least one positive result. Further rollout of pharmacogenomic testing in the general population, whether commercial or clinic-based, could have a far-reaching impact given the likelihood of identifying atypical variants in most individuals.

Fewer than $6 \%$ of participants changed a prescription medication in response to their PGT results 6 months after testing, and $<1 \%$ reported doing so without consulting a health-care provider. A 2012 cross-sectional, posttesting survey of PGT customers from three companies ${ }^{6}$ found similar self-reported rates of medication changes: among 1,048 customers, $4.4 \%$ reported changing a prescription medication and $0.4 \%$ reported doing so without consulting a physician. Although the infrequency of medication changes made without provider consultation is encouraging, it should be reiterated that the definition of "health-care provider" was not specified; therefore, some participants may have reported interactions with nonprescribing professionals (e.g., nurses or alternative medicine practitioners).

Here, the number of atypical pharmacogenomic results received was associated with the probability of changing a prescription medication post-PGT; however, participants' explanations of what prompted these changes made clear that other PGT results (e.g., genetic risk estimate for coronary heart disease) and post-PGT follow-up care (e.g., serum cholesterol testing) also played a role. Future evaluations of the impact of DTC-PGT on prescription medication should consider the broader testing experience, including nonpharmacogenomic results and any clinical follow-up that may lead to changes to a consumer's prescription medication regimen.

The potential utility of pharmacogenomic results is limited by the pharmacological treatment needs of a particular individual at a particular time. This may explain, in part, why so few participants-relative to the $91.2 \%$ receiving a positive pharmacogenomic result and to the $49.0 \%$ who predict using their results to guide future medication decisions-reported changing a prescription medication in our study. Moreover, despite inclusion of pharmacogenomic information on an increasing number of product inserts, use of genetic testing to guide medication selection and dosing remains limited. ${ }^{20}$ Why participants did or did not believe that they would use their pharmacogenomic results to guide future use of medication is unclear, although participants who thought they would use their results in the future had more frequently received an atypical drug response result and more frequently reported already having made a change in medication by 6 -month follow-up. Multiple explanations for these trends are plausible: consumers may believe that atypical response results are inherently more "actionable" than typical response results; consumers who have 
Table 5 Logistic regression of specific medication changes reported post-PGT on number of atypical response pharmacogenomic results received $(N=961)$

\begin{tabular}{lccc} 
& Events, $\boldsymbol{n}$ & Univariable OR (95\% Cl) $\boldsymbol{P}$ value & Multivariable $^{\mathbf{a}}$ \\
\hline Started taking a new medication & 13 & $2.11(1.29,3.44) 0.003$ & $2.01(1.18,3.42) 0.01$ \\
Stopped taking a current medication & 28 & $1.62(1.15,2.29) 0.005$ & $1.48(1.02,2.16) 0.04$ \\
Increased the dose of a current medication & 5 & $0.53(0.20,1.39) 0.19$ & $0.46(0.16,1.32) 0.15$ \\
Decreased the dose of a current medication & 13 & $2.11(1.29,3.44) 0.003$ & $1.94(1.14,3.30) 0.02$ \\
Switched from one medication to another & 15 & $2.31(1.46,3.66)<0.001$ & $2.10(1.24,3.54) 0.006$ \\
\hline
\end{tabular}

$\mathrm{Cl}$, confidence interval; OR, odds ratio; PGT, personal genomic testing.

aMultivariable models adjusted for age, gender, Hispanic ethnicity, self-reported health, and PGT company.

already made some change prompted by their results may be more optimistic about their future potential; or certain consumers may be more likely to engage with and utilize their PGT results in managing their own health care, both immediately following testing and in the future, due to personal characteristics or their original motivations for seeking PGT.

In a recent study of DTC pharmacogenomic testing, Bloss et al. reported that, compared with participants who had not yet received their pharmacogenomic results, those who had received them reported more physician visits, higher rates of sharing their results with a physician, and more physicianordered follow-up services. ${ }^{8}$ Although Bloss et al. did not attempt to explain why receipt of pharmacogenomic information prompts greater health services usage in their study, they suggested that pharmacogenomic information may be more likely than other PGT-derived information (e.g., disease risk estimates) to be added to the medical record (because of its perceived actionability) and therefore more likely to be used in medical decision making. If these explanations are correct, then we might reasonably expect atypical drug response ("red flag") results to more acutely motivate medical interactions and interventions than typical drug response results. Our study, however, which found no association between receipt of atypical pharmacogenomic results and these same health-care outcomes, adds a layer of complexity to this picture. Although receipt of pharmacogenomic information, generally, may lead to increased utilization of health care, the content of those results in fact appears to play a limited role in this effect.

Population characteristics could also explain the lack of observed effect; for example, the mean of nearly seven reported health-care visits in the past year within our sample is higher than expected in the US population, ${ }^{21}$ and the distribution of this variable suggests our estimates are disproportionately influenced by a small number of high-frequency users of health care.

Our study further provides anecdotal evidence of both potential harms and potential benefits to consumers of DTC pharmacogenomic testing. In case 1, a consumer reduces, without physician consultation, her dose of a cholesterol-lowering fibrate in response to a result indicating increased risk of myopathy from statins. The consumer has therefore misinterpreted her PGT results and applied them too broadly; there is currently no evidence from the Pharmacogenomics Knowledgebase $(\mathrm{PharmGKB})^{22}$ of an effect of the statin-related SNPs for which the consumer was tested on drugs in the fibrate class. Given this consumer's reported personal and family history of heart disease, diabetes, and cholesterol, the risk of adverse health outcomes associated with failure to adhere to her cholesterol-lowering medication regimen probably far exceed the probability of statin-induced myopathy (if she were using a statin). ${ }^{23}$

Case 2 highlights the potential for DTC-PGT to democratize access to genetic information ${ }^{24}$ and motivate patient engagement. Here, a consumer and her provider together decided to lower her dose of oral contraceptives in response to an increased risk of deep vein thrombosis with estrogen supplementation. Genetic screening for thrombosis risk prior to prescription of estrogencontaining oral contraceptives is not currently recommended in the absence of a family history due to the low absolute risk of thrombosis, even among those with a genetic predisposition. ${ }^{25}$ Existing guidelines do, however, address incorporation of genetic risk information when already known. In the absence of a family history, women using estrogen-containing oral contraceptives should avoid additional risk factors, such as obesity and smoking; in the presence of a family history, women should avoid estrogen-containing oral contraceptives altogether. ${ }^{26}$ Given that family history of thrombosis is often unknown or unreliable, ${ }^{27}$ and that equally effective low-estrogen and non-estrogen-containing contraceptive options exist, one could argue that regardless of the clinical utility of population screening for thrombosis risk, PGT has, for this consumer, provided a tangible benefit in the form of a patient-physician dialogue and informed decision making regarding the use of estrogen.

Strengths of the PGen Study include the longitudinal collection of data, recruitment of new customers from two leading PGT companies, and incorporation of individual-level genetic information with extensive survey data. Limitations include the potential for selection bias due to the collection of voluntary survey data and the exclusion of participants from certain analyses due to missing data. In addition, our sample may not be typical of the DTC-PGT consumer population circa 2012 because of the recruitment strategies used, particularly for Pathway customers. Users of a health-based social networking site were directly targeted and offered subsidized testing; therefore, we may expect some enrichment of our sample for individuals who typically would not have pursued testing independently because of a lack of awareness or sensitivity to price.

Our post-PGT measures are limited to 6 months of followup, encompass the effects of all PGT-obtained genetic risk 


\section{ORIGINAL RESEARCH ARTICLE}

information (not just pharmacogenomic information), and rely entirely on self-report. These outcome measures could be improved by the incorporation of medical records data. However, because we were particularly interested in determining the frequency of prescription medication changes made without clinician involvement, self-reported data collection was essential. Further, because of the way in which prescription medication changes and physician consultation were measured, we were unable to identify those participants who may have considered making a prescription medication change but consulted with a health-care provider and ultimately decided against making the change. Therefore, the role of the healthcare provider in post-PGT decision making surrounding prescription medications is probably not fully captured in our study.

Finally, our findings may be generalizable to consumers obtaining pharmacogenomic information via DTC-PGT (although it should be noted that as of 2016, neither 23andMe nor Pathway currently offers pharmacogenomic testing via the DTC model), but they are probably not applicable to recipients of clinician-mediated pharmacogenomic testing. PGen Study participants tended to be high-earning, frequent prescription medication users, with high levels of health insurance coverage; therefore, how pharmacogenomic information is used by consumers to self-manage their care may differ in groups without these qualities, particularly among those with low incomes or poor insurance coverage of prescription medications.

In conclusion, receipt of positive pharmacogenomic results via DTC-PGT is associated with posttesting prescription medication changes, but the proportion of consumers who report making such a change during the 6 months following testing is small. Further investigation of how physician consultation during the post-PGT period motivates or discourages such changes is warranted.

\section{SUPPLEMENTARY MATERIAL}

Supplementary material is linked to the online version of the paper at http://www.nature.com/gim

\section{ACKNOWLEDGMENTS}

The PGen Study is supported by the National Institutes of Health (NIH) National Human Genome Research Institute (R01-HG005092). During manuscript preparation, D.A.C. was supported by a Canadian Institutes of Health Research Doctoral Foreign Study Award; she is currently supported by a Michael G. DeGroote Postdoctoral Fellowship from McMaster University and a Canadian Institutes of Health Research Postdoctoral Fellowship. J.L.V. received support from a Harvard KL2/Catalyst Medical Research Investigator Training Award (KL2-TR001100) and is currently supported by Career Development Award IK2 CX001262 from the US Department of Veterans Affairs Clinical Sciences Research and Development Service. C.H.vdW. is supported by K.F. Hein Fonds, De Stichting Jo Kolk Studiefonds Voor Vrouwen, and De Koninklijke Nederlandse Maatschappi Pharmacie Stipendiafonds. Investigators on this project were also supported by NIH U01-HG006500, U19-HD077671, U01-HG008685, R01HG006615, R01-CA154517, P60-AR047782, and U41-HG006834. The content is solely the responsibility of the authors and does not necessarily represent the official views of the National Center for Research Resources, the National Institutes of Health, the Department of Veteran Affairs, or the Canadian Institutes of Health Research.

Nonauthor members of the PGen Study at the time of publication are as follows: Joel B. Krier, Margaret H. Helm, Sarah S. Kalia, Kurt D. Christensen, Lisa S. Lehmann, Harvard Medical School and Brigham and Women's Hospital; Mack T. Ruffin IV, Lan Q. Le, Jenny Ostergren, University of Michigan School of Public Health; Wendy R. Uhlmann, Mick P. Couper, University of Michigan; Joanna L. Mountain, Amy K. Kiefer, 23andMe; Adrian Vilalta, Pathway Genomics; Scott D. Crawford, Survey Sciences Group; L. Adrienne Cupples, Clara A. Chen, Catharine Wang, Boston University; Stacy W. Gray, Dana-Farber Cancer Institute; Barbara A. Koenig, University of California San Francisco; Kimberly Kaphingst, University of Utah; Sarah Gollust, University of Minnesota.

\section{DISCLOSURE}

RCG declares research support from the National Institutes of Health. He has received compensation for advisory services or speaking from Invitae, Prudential, Illumina, Helix, Roche, and Genome Medical, Inc. The other authors declare no conflict of interest.

\section{REFERENCES}

1. McGuire AL, Burke W. An unwelcome side effect of direct-to-consumer personal genome testing: raiding the medical commons. JAMA 2008;300: 2669-2671.

2. Reid RJ, McBride CM, Alford SH, et al. Association between health-service use and multiplex genetic testing. Genet Med 2012;14:852-859.

3. Bloss CS, Schork NJ, Topol EJ. Effect of direct-to-consumer genomewide profiling to assess disease risk. N Engl J Med 2011;364:524-534.

4. Caulfield TA. The informed gatekeeper?: a commentary on genetic tests, marketing pressure and the role of primary care physicians. Health Law Rev 2001;9:14-18.

5. US Food and Drug Administration. Warning letter to Ann Wojcicki, CEO of 23andMe (document no. GEN1300666). 22 November 2013. http://www. fda.gov/ICECl/EnforcementActions/WarningLetters/2013/ucm376296.htm. Accessed 11 June 2014.

6. Kaufman DJ, Bollinger JM, Dvoskin RL, Scott JA. Risky business: risk perception and the use of medical services among customers of DTC personal genetic testing. J Genet Couns 2012;21:413-422.

7. Carere DA, Couper MP, Crawford SD, et al.; PGen Study Group. Design, methods, and participant characteristics of the Impact of Personal Genomics (PGen) Study, a prospective cohort study of direct-to-consumer personal genomic testing customers. Genome Med 2014;6:96.

8. Bloss CS, Schork NJ, Topol EJ. Direct-to-consumer pharmacogenomic testing is associated with increased physician utilisation. J Med Genet 2014;51: 83-89.

9. 23andMe Inc. https://www.23andme.com/. Accessed November 2014.

10. Pathway Genomics Corp. www.pathway.com. Accessed November 2014

11. PatientsLikeMe. www.patientslikeme.com. Accessed 1 September 2015.

12. The American Association for Public Opinion Research. Standard definitions: final dispositions of case codes and outcome rates for surveys, 7th edition. AAPOR; 2011.

13. Lehmann LS, Kaufman DJ, Sharp RR, et al. Navigating a research partnership between academia and industry to assess the impact of personalized genetic testing. Genet Med 2012;14:268-273.

14. White House Office of Management and Budget. Revisions to the Standards for the Classification of Federal Data on Race and Ethnicity. Executive 
Office of the President Office of Management and Budget (OMB) Office of Information and Regulatory Affairs. The White House: Washington, DC, 1997.

15. Ware JE Jr, Gandek B. Overview of the SF-36 Health Survey and the International Quality of Life Assessment (IQOLA) Project. J Clin Epidemiol 1998;51: 903-912.

16. Ritter PL, Stewart AL, Kaymaz H, Sobel DS, Block DA, Lorig KR. Self-reports of health care utilization compared to provider records. J Clin Epidemiol 2001;54:136-141.

17. Dubay LC, Lebrun LA. Health, behavior, and health care disparities: disentangling the effects of income and race in the United States. Int J Health Serv 2012;42:607-625.

18. Kennedy J, Morgan S. A cross-national study of prescription nonadherence due to cost: data from the Joint Canada-United States Survey of Health. Clin Ther 2006;28:1217-1224.

19. Van Driest SL, Shi Y, Bowton EA, et al. Clinically actionable genotypes among 10,000 patients with preemptive pharmacogenomic testing. Clin Pharmacol Ther 2014;95:423-431.
20. Gillis NK, Innocenti F. Evidence required to demonstrate clinical utility of pharmacogenetic testing: the debate continues. Clin Pharmacol Ther 2014;96:655-657.

21. National Center for Health Statistics. Health, United States, 2015: With Special Feature on Racial and Ethnic Health Disparities. Hyattsville, MD, 2016.

22. Thorn CF, Klein TE, Altman RB. PharmGKB: the Pharmacogenomics Knowledge Base. Methods Mol Biol 2013;1015:311-320.

23. Stewart A. SLCO1B1 Polymorphisms and Statin-Induced Myopathy. PLoS Curr 2013;5:.

24. Foster MW, Sharp RR. Out of sequence: how consumer genomics could displace clinical genetics. Nat Rev Genet 2008;9:419.

25. Vandenbroucke JP, van der Meer FJ, Helmerhorst FM, Rosendaal FR. Factor $V$ Leiden: should we screen oral contraceptive users and pregnant women? BMJ 1996:313:1127-1130.

26. Swen JJ, Nijenhuis $M$, de Boer $A$, et al. Pharmacogenetics: from bench to byte-an update of guidelines. Clin Pharmacol Ther 2011;89:662-673.

27. Cushman M. Thrombophilia testing in women with venous thrombosis: the 4 P's approach. Clin Chem 2014;60:134-137. 